\title{
Survivin Downregulation Is Associated with Vasectomy-Induced Spermatogenic Damage and Apoptosis
}

\author{
May Al-Maghrebi ${ }^{a}$ Elijah O. Kehinde ${ }^{b}$ Jehoram T. Anim ${ }^{c}$ \\ Departments of ${ }^{a}$ Biochemistry, ${ }^{\text {b }}$ Surgery (Division of Urology) and ${ }^{C}$ Pathology, Faculty of Medicine, \\ Kuwait University, Safat, Kuwait
}

\section{Key Words}

Survivin · Vasectomy $\cdot$ Apoptosis $\cdot$ Spermatogenesis

\begin{abstract}
Objective: To evaluate the expression of the apoptotic genes survivin, Bax and $\mathrm{Bcl}-2$ in vasectomized rabbits and to determine their relation with vasectomy-induced spermatogenic impairment and germ cell apoptosis. Materials and Methods: Twelve adult rabbits (6-12 months old) were divided into three groups: sham control, unilateral vasectomy or bilateral vasectomy. Six months after vasectomy, testicular tissue was analyzed for germ cell apoptosis and DNA fragmentation by the TUNEL assay and gel electrophoresis, respectively. Spermatogenesis was assessed using the Johnsen score. The relative gene expression of survivin, $\mathrm{Bax}$ and $\mathrm{Bcl}-2$ was measured using reverse transcription followed by realtime PCR. Results: Compared to sham animals, a significant decrease in testicular survivin mRNA levels was measured in the two vasectomy animal groups $(p<0.05)$. This was accompanied by a significant increase in the $\mathrm{Bax}: \mathrm{Bcl}-2$ ratio in the vasectomized animals ( $p<0.05$ ). In addition, these data showed positive correlation with enhanced apoptotic index, damage to spermatogenesis and DNA fragmentation after vasectomy. Conclusion: These findings demonstrate that vasectomy-induced damage to spermatogenesis due to testicular apoptosis may be associated with survivin downregulation and Bax overexpression.
\end{abstract}

Copyright $\odot 2011$ S. Karger AG, Basel

\section{KARGER \\ Fax +4161306 1234 \\ E-Mail karger@karger.ch}

www.karger.com
(C) 2011 S. Karger AG, Basel

1011-7571/11/0205-0449\$38.00/0

Accessible online at:

www.karger.com/mpp

\section{Introduction}

Worldwide, vasectomy is the most relied upon contraceptive method among an estimated 33 million male partners aged 15-49 [1]. In the United States approximately half a million vasectomies are performed each year. Postvasectomy complications such as hematoma, infections or orchalgia are infrequent and minor and do not require hospitalization or serious medical management. Vasectomy is therefore a popular, safe and costeffective method of contraception.

Apoptosis plays a prominent role in human male infertility, which could be caused by either increased or decreased rate of apoptosis since both will lead to disrupted testicular homeostasis [2]. In states of impaired sperm production, the formation of intratesticular apoptotic bodies has been observed with implication of several factors of the apoptotic machinery in the process [3]. Thus, an interest was instigated to explore the molecular determinants that control germ cell apoptosis and their signaling pathways. One of the well-known survival-promoting genes is survivin, an inhibitor of apoptosis. Its role in regulating cellular proliferation, cell cycle and programmed cell death made it a good candidate gene for regulating germ cell apoptosis [4]. One of the early indications of its possible role in human spermatogenesis came from a study by Weikert et al. [5]. The group demonstrated that survivin is downregulated in spermato-

Dr. May Al-Maghreb

Department of Biochemistry, Faculty of Medicine

Kuwait University, PO Box 24923

13110 Safat (Kuwait)

Tel. +965 2498 6447, E-Mail malmaghrebi@ @sc.edu.kw 
genic disorders, which implicated it as a regulatory factor in germ cell proliferation and apoptosis with a possible role in human spermatogenesis. Other reports also suggested a role of survivin in infertility-related processes like its involvement in ensuring optimal development of bovine blastocysts and suppression of apoptosis of preimplantation embryos [6] and increasing the chance of patients to get pregnant during in vitro fertilization [7].

Against this background we opted to compare the gene expression of survivin and evaluate its biological significance in vasectomy-induced apoptotic germ cell death in a rabbit model. We also aimed to establish whether there is a correlation between the gene expression of survivin, Bax and Bcl-2 and impaired spermatogenesis 6 months after vasectomy.

\section{Materials and Methods}

\section{Experimental Animal Model}

Six- to twelve-month-old New Zealand White rabbits were used. They were cared for according to the guidelines of the $\mathrm{Ku}$ wait University Animal Research Center. All experiments were performed under anesthesia using intravenous ketamine and $x y-$ alzine mixture (Sigma-Aldrich Co. Ltd., Pool, Dorset, UK). Three animal groups (4 animals/group) were studied: group A animals (sham, control group) were subjected to left scrotal incisions with exposure of the testis and the wounds were closed using 3/0 chromic catgut (CCG) suture (Ethicon Ltd., Edinburgh, UK). Group $B$ animals underwent unilateral vasectomy whereby $4 \mathrm{~cm}$ of the left vas deferens was excised and the ends of the vas were tied with $3 / 0$ silk sutures. The wound was closed with 3/0 CCG suture. Group $\mathrm{C}$ animals had a bilateral vasectomy during which $4 \mathrm{~cm}$ of the vas deferens (left and right) was excised and the ends were tied with 3/0 silk sutures. The wounds were closed with 3/0 CCG suture. The right testis served as a positive internal control in animals in groups A and B. Six months after vasectomy, the two testes were excised under anesthesia. The harvested testis was divided into four equal parts and each quarter used as follows: assessment of testicular histology using Johnsen's score, TUNEL assay, gene expression measurement and evaluation of DNA fragmentation.

\section{Histological Examination}

The mean testicular biopsy score (Johnsen score) was used to assess morphological damage of testicular tissue caused by vasectomy using hematoxylin and eosin staining [8]. Testicular histology was assessed blindly for both right and left testes of the three experimental groups of animals. Any other important histological features were noted. The Johnsen score is based on the premise that with testicular damage, there is successive disappearance of the most mature cell type, with progressive degeneration of germinal epithelium: first, spermatozoa and spermatids, then spermatocytes and finally Sertoli's cells disappear in that order. For experimentally induced damage to the testis, this premise has been found to be largely correct [9].
TUNEL Assay

The TUNEL assay was performed using the in situ Cell Death Detection Kit according to the manufacturer's protocol (Roche Applied Sciences, Mannheim, Germany). Briefly, harvested testicular tissues were fixed and digested with $100 \mu \mathrm{g} / \mathrm{ml}$ Proteinase K (Roche Applied Sciences, Foster City, Calif., USA). Permeabilized samples were incubated with the TUNEL reaction mixture that contains TdT and fluorescein-dUTP. During incubation, TdT catalyzes the addition of fluorescein-dUTP at free $3^{\prime}-\mathrm{OH}$ groups in single- and double-stranded DNA. After washing, the label incorporated at the damaged sites of the DNA was marked by an antifluorescein antibody conjugated with the reporter enzyme peroxidase. Immune complex visualization and stained cell analysis was done by light microscopy. The number of apoptotic cells was evaluated within the seminiferous tubules of the testes using the following simplified system: a score of $<1$ indicates few tubules involved with up to 1 apoptotic cell/tubule; a score of $1+$ indicates scattered tubules involved with $<4$ apoptotic cells present per tubule; a score of $2+$ indicates several tubules involved with 4-6 apoptotic cells present per tubule, and a score of $3+$ indicates many tubules involved with $>6$ apoptotic cells present per tubule.

\section{RNA Extraction, Reverse Transcription and Real-Time PCR}

Total RNA was purified from freshly isolated testes from control and experimental animals using TRIzol (Invitrogen, Carlsbad, Calif., USA) according to the manufacturer's instructions. Purified RNA samples were incubated with DNase I to remove contaminating DNA molecules. Reverse transcription was performed using $1 \mu \mathrm{g}$ of total RNA using random hexamers. Specific Taqman primers and probes were used to detect the gene expression of survivin (forward primer: $5^{\prime}$-GGCCCAGTGTTTCTTCTGCTT-3', reverse primer: 5'-CAACCGGACGAATGCTTTTT-3' and Taqman probe: 5'-AGCCAGATGACGACCCCATAGAGGAACA-3'), Bcl-2 (forward primer: 5'-GGACGCGAAGTGCTATTGGT-3', reverse primer: 5'-TCACGATCTCCCGGTTATCATA-3' and Taqman probe: 5'-CCGGAAGGATGGCGCAAGCC- $3^{\prime}$ ) and Bax (forward primer: $5^{\prime}$-TGACATGTTTTCTGATGGCAACT-3', reverse primer: 5'-GGTGCACAGGGCCTTGAG-3' and Taqman probe: 5'-TGTTGCCCTCTTCTACTTTGCCAGCAA-3') by real-time PCR using the 7000 Sequence Detection System from Applied Biosystems (Carlsbad, Calif., USA).

The expression of the housekeeping gene $\beta$-actin was measured in all samples to normalize for sample-to-sample differences in RNA input, quality, and reverse transcription efficiency. The relative expression of mRNA species was calculated by the comparative $\Delta \mathrm{C}_{\mathrm{T}}$ method.

\section{Evaluation of DNA Fragmentation}

Portions of excised testicular tissue were snap-frozen in liquid nitrogen, powdered and stored at $-80^{\circ} \mathrm{C}$. Subsequently, these tissues were subjected to extraction of genomic DNA. Briefly, cells were lysed in a hypotonic lysis buffer $(10 \mathrm{~mm}$ Tris-HCI, $1 \mathrm{~mm}$ EDTA, $0.2 \%$ Triton X-100, pH 7.5) followed by centrifugation. Isolated DNA was incubated in a proteinase $\mathrm{K}$ solution at $37^{\circ} \mathrm{C}$ for $12 \mathrm{~h}$ and precipitated with isopropanol at $-20^{\circ} \mathrm{C}$. Equal DNA concentrations (10 $\mu \mathrm{g} / \mathrm{lane})$ were loaded and run on a $1.0 \%$ agarose gel containing $0.001 \%$ ethidium bromide and then visualized under ultraviolet light. 
Table 1. Histological analysis and TUNEL assay

\begin{tabular}{llllll}
\hline Animal group & \multicolumn{2}{l}{ Histological analysis } & & \\
\cline { 2 - 3 } & \multicolumn{2}{l}{ Johnsen score } & & & apoptotic score \\
\cline { 2 - 3 } \cline { 5 - 6 } & right testis & left testis & & right testis & left testis \\
\hline Control sham & $9.67 \pm 0.57$ & $9 \pm 0$ & & $1+$ & $1+$ \\
Unilateral left vasectomy & $10 \pm 0$ & $6.7 \pm 1.35^{1}$ & & $<1$ & $2+$ \\
Bilateral vasectomy & $4.33 \pm 0.57^{1}$ & $4 \pm 1.57^{1}$ & & $3+$ & $2+$ \\
\hline
\end{tabular}

Values are expressed as means \pm standard deviation from 4 animals per group.

${ }^{1} \mathrm{p}<0.05$ is considered significant data as compared to sham levels.

\section{Measurement of Serum Level of Sex Steroid Hormones}

Five milliliters of blood was obtained from the ear veins in plain tubes at $0 \mathrm{~h}$ and at 24 weeks. Serum was separated using standard laboratory methods and stored at $-80^{\circ} \mathrm{C}$. From each serum sample, the levels of the following hormones were determined using ELISA technique: luteinizing hormone (LH), follicle-stimulating hormone (FSH) and dihydrotestosterone (DHT). The ELISA kits were purchased from Diagnostic System Laboratories, Inc. (Brea, Calif., USA). The absorbance of the immunoreactions was measured using a Sunrise microplate reader (Tecan, Männedorf, Switzerland) set to dual wavelength measurement at $450 \mathrm{~nm}$ with background wavelength correction set at $620 \mathrm{~nm}$.

\section{Statistical Analysis}

The data was analyzed using the Statistical Package for the Social Sciences (SPSS) version 13. Comparison between groups was done by Student's t test; $\mathrm{p}<0.05$ was considered statistically significant. Morphological assessment was expressed as a mean $( \pm$ standard error of the mean) of the Johnsen testicular biopsy score.

\section{Results}

\section{Assessment of Germ Cell Apoptosis}

The mean testicular biopsy scores (Johnsen score) for testes subjected to either unilateral (group B) or bilateral (group C) vasectomies are shown in table 1 and illustrated in figure $1 \mathrm{~A}-\mathrm{E}$. The histological features showed significant damage in group $C(p<0.05)$ and less severe damage in group $B(p<0.05)$ compared to sham, indicating the damaging long-term effect of the surgical procedure on the viability of testicular germ cells. Furthermore, cellular apoptosis was detected using the TUNEL assay. Positive germ cell apoptosis was scored using the described scoring system in 'Materials and Methods' and histologically represented in figure $1 \mathrm{~F}-\mathrm{J}$. Increased scores of apoptotic cell death corresponded more with the damaged testicular tissues from groups $\mathrm{B}$ and $\mathrm{C}$ in comparison to the sham control group A (table 1).

\section{Effect of Vasectomy on the Gene Expression of} Apoptosis Genes

mRNA levels of all three apoptosis genes and the housekeeping gene $\beta$-actin were detected in all testicular tissue samples. The calculated relative gene expression values are shown in table 2 . Survivin expression was significantly downregulated $(\mathrm{p}<0.05)$ in animals with unilateral (left testis only) and bilateral vasectomies. Bax expression was significantly upregulated in the two vasectomy groups compared with the sham control $(\mathrm{p}<0.05)$, while Bcl-2 expression levels were not significantly changed after vasectomy ( $p>0.05)$. Accordingly, the calculated ratio of Bax to $\mathrm{Bcl}-2$ relative gene expression showed a significant increase $(\mathrm{p}<0.05)$ in both vasectomy animal groups.

\section{Hormonal Assessment}

There were no significant changes in the hormonal levels before and after the vasectomy procedures (table 3 ).

\section{Evaluation of DNA Fragmentation after Vasectomy}

Significant DNA fragmentation, shown as a distinctive DNA ladder, was observed in animals subjected to bilateral vasectomy and to a lesser degree in those with unilateral vasectomy while it was absent in control samples as shown in figure 2 .

\section{Discussion}

In the present study, we have looked at the gene expression of survivin, an inhibitor of apoptosis, to determine its involvement in the long-term vasectomy-induced spermatogenic damage due to germ cell apoptosis. The use of the 6-month period after vasectomy was based on preliminary findings from a study done by our group [9], 

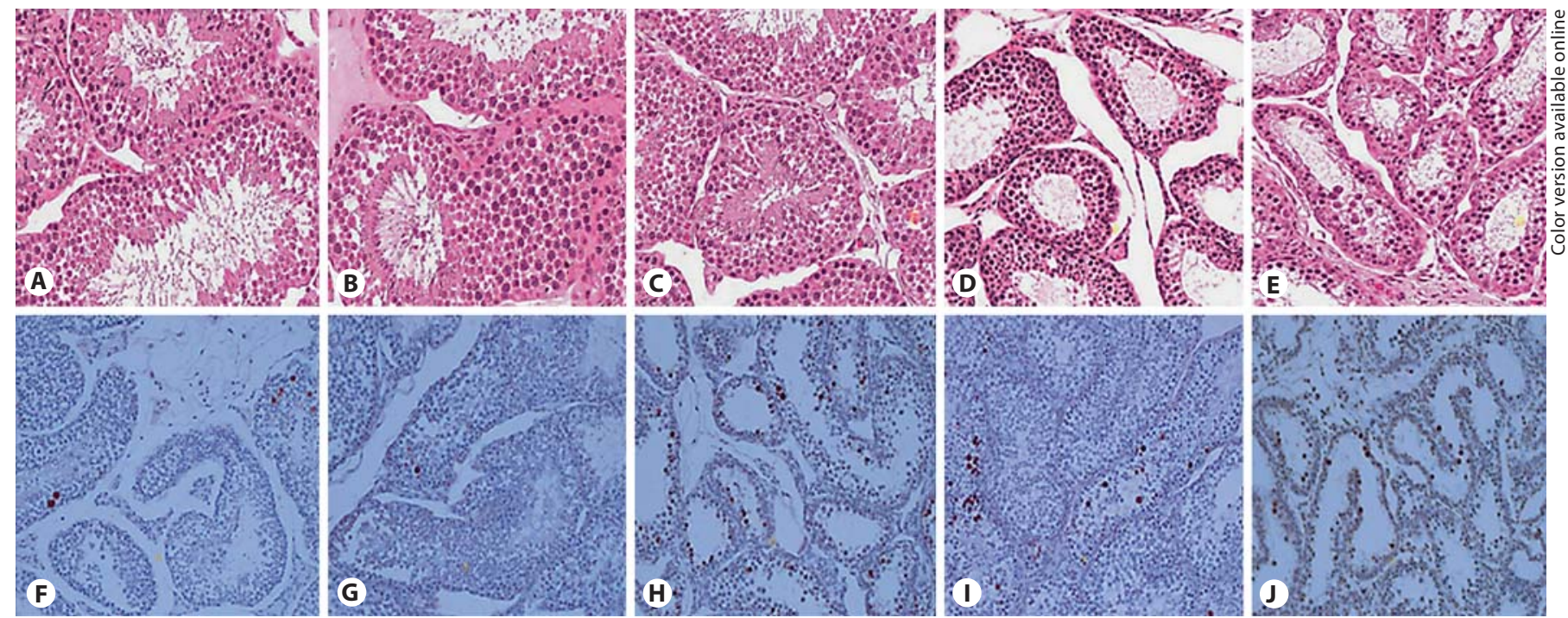

Fig. 1. Histological evaluation of testicular biopsy. A-E Johnsen score. Sham control: score 9 (A), score 9.67 (B); unilateral vasectomy: score 10 (C), score 7 (D); bilateral vasectomy: score 4 (E). F-J Apoptosis score. Sham control: score 1+ (F); unilateral vasectomy: score <1 (G), score 2+ (H); bilateral vasectomy: score 3+ (I), score 2+ (J).

Table 2. Relative expression of apoptosis genes

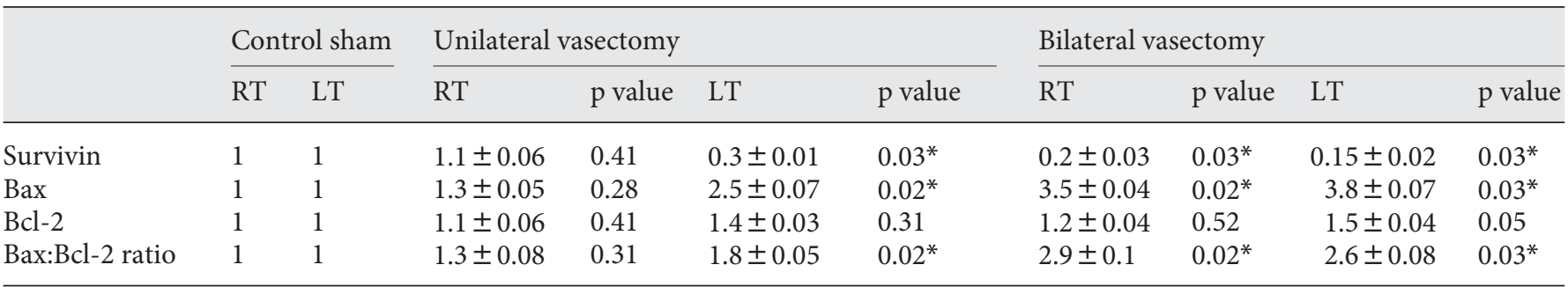

Values are expressed as means \pm standard error of the mean from 4 animals per group. RT $=$ Right testis; LT $=$ left testis.

${ }^{*} \mathrm{p}<0.05$ is considered significant data as compared to sham levels.

Table 3. Effect of vasectomy on serum levels of LH, FSH and DHT

\begin{tabular}{|c|c|c|c|c|c|c|c|c|c|}
\hline & \multicolumn{3}{|c|}{ Control sham } & \multicolumn{3}{|c|}{ Unilateral vasectomy } & \multicolumn{3}{|c|}{ Bilateral vasectomy } \\
\hline $\mathrm{FSH}, \mathrm{mIU} / \mathrm{ml}$ & $1.9 \pm 0.04$ & $1.9 \pm 0.01$ & 0.41 & $1.9 \pm 0.04$ & $1.8 \pm 0.05$ & 0.34 & $1.8 \pm 0.03$ & $1.81 \pm 0.02$ & 0.9 \\
\hline DHT, pg/ml & $171 \pm 1.5$ & $175 \pm 1.95$ & 0.14 & $171 \pm 1.8$ & $176 \pm 1.7$ & 0.14 & $167 \pm 1.1$ & $166 \pm 3.1$ & 1.0 \\
\hline
\end{tabular}

Values are expressed as means \pm standard error of the mean from 4 animals per group. 
which showed that following experimental torsion of testis or vasectomy, changes at the molecular and cellular levels continue to take place in the testis for about 3-6 months.

A positive correlation was established in this study between the downregulation of survivin gene expression and the increase in apoptosis and the degeneration of the germinal epithelium. Survivin expression was decreased in testicular tissues from animals with unilateral and bilateral vasectomy $(\mathrm{p}<0.05)$. Decreased survivin mRNA levels also coincided with an increased apoptotic score $(\mathrm{p}<0.05)$ as demonstrated by the TUNEL assay, which further supports the role of survivin as an inhibitor of apoptosis and in this case germ cell apoptosis. Although there have been no reports about the role of survivin in vasectomy-induced germ cell apoptosis and impaired spermatogenesis, several studies have looked at its role in germ cell production. Survivin gene expression was found to be altered in certain spermatogenic disorders. The studies of Weikert et al. [5] have shown that testicular survivin levels in infertile patients were related inversely to the severity of spermatogenic failure $(\mathrm{p}<$ 0.001 ), with a lack of expression in most specimens with premeiotic spermatogenic arrest and in all those with germ cell aplasia $[10,11]$. Another study by Roshdy and Mostafa [12] showed that seminal survivin was significantly high in fertile control males in comparison with oligo-asthenozoospermic and nonobstructive azoospermia patients. Survivin expression was undetectable in individuals with obstructive azoospermia. This further supports a potential role of survivin in male gametogenesis and its possible involvement in determining male fertility.

Another apoptosis marker is the Bax (an apoptosis promoter) to $\mathrm{Bcl}-2$ (an apoptosis inhibitor) ratio. The modulation of cellular levels of these proteins in response to the onset or regression of apoptosis has established them as a reliable apoptosis 'rheostat' for the cell. In our study, Bax levels were significantly higher $(\mathrm{p}<0.05)$ after both surgical procedures in contrast to the Bcl-2 gene expression, which is in agreement with other studies using testicular tissue from animal models and vasectomized men. Testicular biopsies from vasectomized men $(>5$ years) showed reduced sperm yields after vasectomy which correlated with increased apoptosis through the Fas-FasL and Bax pathways and increased DNA fragmentation [13]. In vasectomized rats, damage to spermatogenesis was associated with increased p53, increased Bax expression and germ cell apoptosis more than 8 weeks after vasectomy [14]. The role of Bax in regulating apop-

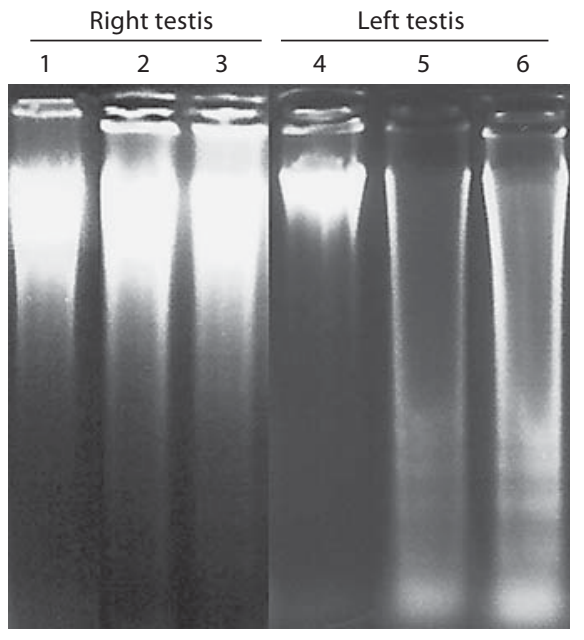

Fig. 2. Detection of DNA fragmentation in testicular tissue. $10 \mu \mathrm{g}$ of purified genomic DNA was separated on $1.0 \%$ agarose gel electrophoresis. Lanes 1-3 represent the right testis, while lanes 4-6 represent the left testis. From left to right: sham control (lanes 1 and 4), unilateral left vasectomy (lanes 2 and 5) and bilateral vasectomy (lanes 3 and 6). The right testis served as the internal positive control for the sham control and unilateral left vasectomy.

tosis in the testis is hypothesized to occur via association with other Bcl-2 family members, Bak and Bcl-w, followed by their translocation into the mitochondria to trigger the release of cytochrome $\mathrm{C}$ and induce the mitochondrial apoptosis pathway [15].

Since spermatogenesis is mainly controlled by the endocrine effect of FSH and the paracrine effect of testosterone, the observed vasectomy-induced testicular dysfunction could also have been a result of an endocrine disruption. Although some studies reported increased levels of FSH, LH and testosterone in men 1 year after vasectomy $[16,17]$, other studies found that there were no significant changes in the levels of these hormones after vasectomy $[18,19]$. In our study, serum levels of FSH, LH and DHT did not show any serum level changes 6 months after vasectomy (table 3).

\section{Conclusions}

Our data indicate that germ cell apoptosis after vasectomy is associated with survivin downregulation. Whether survivin expression is regulated through the mitochondrial pathway or another parallel death-signaling pathway leading to germ cell apoptosis needs to be 
established by further studies. The involvement of survivin in the spermatogenic damage induced by vasectomy identifies another player in germ cell apoptosis and adds to our understanding of the mechanism(s) causing male infertility.

\section{Acknowledgments}

This study was supported by Kuwait University Research grant MB01/05. The authors would like to acknowledge Ms. Reeba Eapin, Dr. Asa Prasad, Ms. Jeena John and Mr. Mathew Abraham for their technical support.

\section{References}

$>1$ Pile JM, Barone MA: Demographics of vasectomy - USA and International. Urol Clin North Am 2009;36:295-305.

-2 Kierszenbaum AL: Apoptosis during spermatogenesis: the thrill of being alive. Mol Reprod Dev 2001;58:1-3.

$>3$ Cagan RL: Spermatogenesis: borrowing the apoptotic machinery. Curr Biol 2003;13: R600-R602.

4 Guha M, Altieri DC: Survivin as a global target of intrinsic tumor suppression networks. Cell Cycle 2009;8:2708-2710.

$\checkmark 5$ Weikert S, Schrader M, Muller M, Krause H, Miller K: Expression of the apoptosis inhibitor survivin in testicular tissue of infertile patients. Int J Androl 2004;27:161-165.

$\checkmark 6$ Fujino K, Yamashita Y, Hayashi A, Asano M, Morishima S, Ohmichi M: Survivin gene expression in granulosa cells from infertile patients undergoing in vitro fertilization-embryo transfer. Fertil Steril 2008;89:60-65.

$>7$ Park SY, Kim EY, Jeon K, Cui X, Lee WD, Kim N, Park SP, Lim JH: Survivin acts as anti-apoptotic factor during the development of bovine pre-implantation embryos. Mol Reprod Dev 2007;74:582-590.

$>8$ Johnsen SG: Testicular biopsy score count - a method for registration of spermatogenesis in human testes: normal values and results of 335 hypogonadal males. Hormones 1970;1: $2-25$. $\checkmark 9$ Kehinde EO, Anim JT, Mojiminiyi OA, AlAwadi F, Shihab-Eldeen A, Omu AE, Fatinikun T, Prasad A, Abraham M: Allopurinol provides long-term protection for experimentally induced testicular torsion in a rabbit model. BJU Int 2005;96:175-180.

10 Weikert S, Schrader M, Christoph F, Schulze W, Krause H, Müller M, Miller K: Quantification of survivin mRNA in testes of infertile patients and in testicular germ cell tumours: high levels of expression associated with normal spermatogenesis. Int J Androl 2005;28: 224-229.

11 Weikert S, Schrader M, Müller M, Schulze W, Krause H, Miller K: Expression levels of the inhibitor of apoptosis survivin in testes of patients with normal spermatogenesis and spermatogenic failure. Fertil Steril 2005;83: 1100-1105.

12 Roshdy N, Mostafa T: Seminal plasma survivin in fertile and infertile males. J Urol 2009;181:1269-1272.

13 O'Neill DA, McVicar CM, McClure N, Maxwell P, Cooke I, Pogue KM, Lewis SEM: Reduced sperm yield from testicular biopsies of vasectomized men is due to increased apoptosis. Fertil Steril 2007;87:834-841.

14 Shiraishi K, Naito K, Yoshida K: Vasectomy impairs spermatogenesis through germ cell apoptosis mediated by the p53-Bax pathway in rats. J Urol 2001;166:1565-1571.
15 Yan W, Samson M, Jégou B, Toppari J: Bcl-w forms complexes with Bax and Bak, and elevated ratios of $\mathrm{Bax} / \mathrm{Bcl}-\mathrm{w}$ and $\mathrm{Bak} / \mathrm{Bcl}-\mathrm{w}$ correspond to spermatogonial and spermatocyte apoptosis in the testis. Mol Endocrinol 2000;14:682-699.

16 Mo ZN, Huang X, Zhang SC, Yang JR: Early and late long-term effects of vasectomy on serum testosterone, dihydrotestosterone, luteinizing hormone and follicle-stimulating hormone levels. J Urol 1995; 154:2065-2069.

17 Smith K, Tcholakian R, Chowdhury M, Steinberger E: An investigation of plasma hormone levels before and after vasectomy. Fertil Steril 1976;27:144-151.

18 Naik V, Thakur A, Sheth A, Joshi U, Rao S, Pardani D, Kulsreshtha J, Handa R: The effect of vasectomy on pituitary-gonadal function in men. J Reprod Fertil 1976;48:441442.

19 McVicar CM, O’Neill DA, McClureN, Clements B, McCullough S, Lewis SEM: Effects of vasectomy on spermatogenesis and fertility outcome after testicular sperm extraction combined with ICSI. Hum Reprod 2005;20: 2795-2800. 